\title{
Editorial: Special Issue on Robot Learning by Observation, Demonstration and Imitation
}

\author{
Yiannis Demiris and Aude Billard
}

Index Terms-Learning by Observation, Programming by Demonstration (PbD), Learning by Imitation,

\section{INTRODUCTION TO THE SPECIAL ISSUE}

$\mathbf{T}$ HIS special issue addresses issues in Robot Learning by Observation, Demonstration and Imitation, topics at the heart of an international Symposium on Imitation organised during AISB in Hertforshire in 2005. The special issue contains selected extended contributions both from the symposium participants and the wider academic community following a public call for papers in 2006.

In their paper, Alissandrakis, Nevahiv and Dautenhahn focus on a key problem in imitation, that of body mapping when the demonstrator and the imitator might not share the same embodiment. They approach the problem from a mathematical perspective, formalising body mappings using "correspondence matrices" which allows them to capture different types of associations between degrees of freedom across dissimilarly embodied agents.

Calinon, Guenter and Billard in their paper present a programming by demonstration architecture that extracts the relevant features of a given task and is able to generalise the acquired knowledge to different contexts. The architecture is demonstrated on a humanoid robot learning manipulatory tasks following observation of a human demonstrator.

Belardinelli, Pirri and Carbone present a methodology for learning gaze shifts based on imitation of the gaze shifts of a human demonstrator, wearing a specially designed "gazemachine". This device allows the robot to measure gaze shifts and fixations through mutual vergence, and subsequently generalises the acquired data by learning the scene's salient features and the way saccadic programming is attained.

Wood and Bryson present a paper that addresses skill learning in the domain of a real time computer game where the imitator agent learns how to perform certain tasks within the game's environment following observation of an expert player. The agent learns what contexts are relevant to selecting appropriate actions, what sort of actions are likely to solve a problem, which actions are appropriate in which context, and how to order perception-action pairings when more than on is applicable.

Pardowitz et al in their paper present a system that records, inteprets and reasons over demonstrations of household tasks in an incremental manner. A model-based approach for representing manipulation tasks forms the basis for reasoning about the acquired task knowledge, condensing and interconnecting the acquired data, with the aim of producing more general task knowledge. The acquired data are fused with speech comments acquired during the execution of the task.
Lopes and Santos-Victor in their paper present a developmental strategy for acquiring the capability to learn by imitation. Their system follows a developmental pathway that comprises of three levels, first concentrating on sensory-motor corrodination, and subsequently on world interaction, and imitation. They demonstrate their strategy on a humanoid robot presenting results on the acquisition of perceptual and motor skills.

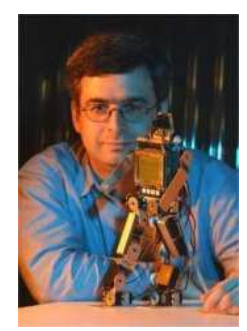

Yiannis Demiris is a Lecturer and head of the Bioinspired Autonomous Robots Team (BioART) at the Intelligent Systems and Networks section (ISN) of the department of Electrical and Electronic Engineering at Imperial College London, which he joined in 2001. He graduated with a BSc (Honours, 1994) from the Department of Artificial Intelligence at the University of Edinburgh, where he also received his $\mathrm{PhD}$ in Intelligent Robotics (1999)), after working as a software engineer at DEC (1994-1995), and a visiting researcher at AIST, Japan (1996-1997)). Dr Demiris' research has made contributions in learning by imitation, the modelling of mirror neurons and their properties, predictive control of attention during human action perception, perspective taking, and developmental approaches to learning visual, motor and cognitive skills. Dr Demiris' current research interests also include the application of action perception to the development of intelligent assistive wheelchairs for children with disabilities, and the perception and control of the actions of groups of agents. Dr Demiris can be contacted at the Department of Electrical and Electronic Engineering, Imperial College London, Exhibition Road, London, SW7 2BT, UK Email: y.demiris@imperial.ac.uk, WWW: http://www.iis.ee.ic.ac.uk/yiannis

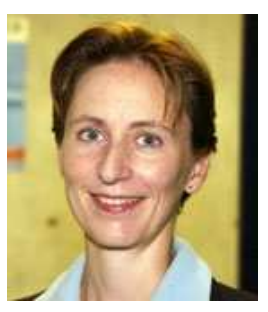

Aude Billard is Associate Professor and head of the Learning Algorithms and Systems Laboratory at the school of Engineering at EPFL (Ecole Polytechnique Fédérale de Lausanne), which she joined in 2002. She received her BSc (1994) and MSc (1995) in Physics from EPFL, with specialization in Particle Physics at the European Center for Nuclear Research (CERN). She received her MSc in Knowledge Based System (1996) and her PhD in Artificial Intelligence (1998) from the department of Artificial Intelligence at the University of Edinburgh. She, then, was a Post-doctoral Fellow at IDSIA and LAMI (EPFL, 1998-99). Aude Billard was a Research Associate (1999-2000), Research Assistant Professor (20002002) and remained an adjunct faculty since then at the Computer Science department at the University of Southern California (USC). Dr. Billard's research interests cover the fields of Artificial Neural Networks, Robotics, Neural Modeling, Computational Neuroscience and more generally Machine Learning. Her work tackles special topics, such as Programming Through Demonstration, Imitation Learning and Language Acquisition. Dr Billard can be contacted at the Learning Algorithms and Systems Laboratory, EPFL, EPFL-STI-I2S-LASA, Station 9, CH1015, Lausanne, Switzerland. Email: aude.billard@epfl.ch, WWW: http://lasa.epfl.ch/ 